Les archives départementales de la Haute-Garonne recèlent un fonds d'archives d'une immense richesse et en grande partie inédit, le fonds de l'ordre de Malte. Celui-ci contient des originaux et des copies, d'époques médiévale et moderne, issus des commanderies templières et hospitalières qui furent réunies dans le Grand Prieuré de Toulouse ${ }^{1}$. La liasse concernant la commanderie de Pézenas recèle deux chartes exceptionnelles qui n'ont pas jusqu'ici attiré l'attention des chercheurs. Il s'agit de deux actes de 1185 et $1202^{2}$ qui sont conservés à l'état d'original et qui sont encore pourvus de leurs sceaux, deux empreintes des vicomtes Trencavel. Pourtant, ces pièces sont loin d'avoir échappé à toute forme de récolement : elles sont mentionnées dans l'Inventaire des sceaux des Archives Départementales de la HauteGaronne réalisé par A. de Font-Réaulx et A. Philippe en 1967-1968³.

Cette récente redécouverte que nous présentons ici permet de réviser la chronologie des empreintes analysées à partir des fonds parisiens et de combler bien des lacunes sur la sigillographie méridionale de la fin du XII ${ }^{\mathrm{e}}$ siècle et du début de la période suivante. Jusqu'à présent, le premier sceau connu d'un membre de la dynastie était celui de Raimond Trencavel, oncle du vicomte Raimond Roger (1194-1209) ${ }^{4}$ : en juillet 1211, il cédait à Simon de Montfort la totalité de ses droits sur l'ensemble des vicomtés qu'il détenait ${ }^{5}$. Or, l'usage du sceau au sein de ce lignage est attesté au moins vingt cinq plus tôt, comme l'indiquaient déjà certains documents provenant du cartulaire familial ${ }^{6}$.

\title{
Les chartes
}

Le 9 décembre 1185, un couple, Pierre de Thézan ${ }^{7}$ et sa femme Guillelma, se retrouva en présence de nombreux témoins dans l'église Saint-Étienne de Boujan ${ }^{8}$ pour échanger des biens (fig. 1, en annexe). Pierre donnait à son épouse quatre champs situés dans le terminium (terroir) d'Aspiran'. Les confronts nous apprennent que le premier champ jouxte le Taurou, affluent de l'Orb, le troisième un moulin, et le quatrième le chemin de Thézan à Aspiran. Il s'agit là de riches parcelles où l'irrigation est possible (le premier champ porte le nom évocateur de Campus Grassus), dans un secteur où sont possessionnés des membres de la haute aristocratie régionale. Mentionnée depuis le milieu du $\mathrm{IX}^{\mathrm{e}}$ siècle, la villa d'Aspiran fut en effet détenue par les plus grands : des Hispani fidèles de Charles le Chauve, les vicomtes

\footnotetext{
${ }^{1}$ Sur celui-ci à l'époque moderne, voir Pierre VIDAL, Hospitaliers et templiers en France méridionale : le Grand Prieuré de Toulouse de l'ordre de Malte. Guide de recherches historiques, archivistiques et patrimoniales, CNRS Framespa et Association des Amis des archives de la Haute-Garonne, Toulouse, 2002.

${ }^{2}$ H Malte, Pézenas, 10 et 19.

${ }^{3}$ Notices $n^{\circ} 47$ et $n^{\circ} 48$. L'inventaire des sceaux date la seconde empreinte de février 1213 alors que l'acte porte distinctement la mention suivante : anno ejusdem incarnationis M CC II mense februarii.

${ }^{4}$ L. MACÉ, «Les sceaux des Trencavel au XIII ${ }^{\mathrm{e}}$ siècle : approches et reconsidérations », Bulletin de la société des sciences, arts et belles lettres du Tarn (à paraître en 2004).

${ }^{5}$ H. G. L., t. VIII, col. 611 ; L.-C. DouËT D'ARCQ, Inventaires et documents des Archives de l'Empire. Collection de sceaux, Paris, 1863 , t. I, p. 384, $\mathrm{n}^{\circ} 759$.

${ }^{6} \mathrm{H}$. DÉBAX, «Un cartulaire, une titulature et un sceau : le programme politique du vicomte Roger II (Trencavel) dans les années 1180 », Les cartulaires méridionaux, Béziers, 20-21 septembre 2002 (à paraître).

${ }^{7}$ Thézan-lès-Béziers, commune du canton de Murviel-lès-Béziers (Hérault), à environ $9 \mathrm{~km}$ au nord-ouest de Béziers.

${ }^{8}$ Boujan-sur-Libron, commune du premier canton de Béziers (Hérault), à $4 \mathrm{~km}$ au nord-nord-est de Béziers et $8 \mathrm{~km}$ au sud-est de Thézan.

${ }^{9}$ Aspiran, hameau de la commune de Thézan, dont le terroir s'étend 2-3 km au sud-ouest du castrum, dans une courbe de l'Orb.
} 
de Béziers, puis l'évêque de Béziers ${ }^{10}$. En 1185, les parcelles mitoyennes des quatre champs sont encore aux mains de la bonne aristocratie : ce sont des honneurs de Bérenger de Thézan, d'Ugo de Paulinet, une condamine et un honneur de l'évêque de Béziers.

En échange, Guillelma cède à son mari tous ses droits dans le terminium et la paroisse de Saint-André de Trémoulet ${ }^{11}$. Si les terres de Pierre se situaient dans la zone d'influence des évêques de Béziers, celles de Guillelma sont dans l'orbite des évêques d'Agde. Dans un acte de 1132 qui mettait au clair la séparation des deux menses, Raimond évêque d'Agde avait en effet donné la villa de Trémoulet au chapitre cathédral Saint-Étienne ${ }^{12}$. Cela explique la confirmation de la charte du 9 décembre 1185, faite quelques jours et transcrite au verso du parchemin, confirmation par Pierre Raimond de Montpeyroux, évêque d'Agde, en forme de simple corroboration : l'évêque fait apposer son sceau et souscrire six des chanoines ${ }^{13}$.

Pour effectuer cette mutation sur leurs biens, les deux époux ont dû solliciter l'accord de leurs proches. Pierre de Thézan a obtenu l'assentiment de son seigneur, Bérenger de Thézan et de la femme de celui-ci Ermessinde, car « il tenait son honneur d'eux en fief ». Guillelma a pu effectuer sa part de l'échange grâce « au conseil et à la volonté de Raimond de Nézignan, son oncle », et il est spécifié qu'elle tient certains de ses droits sur Trémoulet de son grandpère, un autre Raimond de Nézignan ${ }^{14}$. Laudatio seigneuriale d'un côté, laudatio parentum de l'autre, l'individu au Moyen Âge est enserré dans un filet de relations et ne peut se défaire librement de ses biens.

Une curiosité paléographique est à noter au milieu de la charte : à l'endroit où Guillelma prend la parole, Et ego Guillelma dono similiter..., un doigt (l'index) pointé à la verticale est dessiné dans la marge de gauche et montre le $E t$ du début de sa phrase (fig. 2, en annexe). Ce geste rappelle étrangement celui exécuté sur son lit de mort par un seigneur de Thézan, cinquante ans auparavant. Ce seigneur, nommé Bérenger, gisait, très malade, à Béziers, en janvier 1134. Il fit alors donation in extremis de tous ses droits à ses fils, en présence de nombreux parents et amis. À la suite de contestations des chanoines de Béziers sur la détention de dîmes qui faisaient partie de cet héritage, un bayle vicomtal fit une enquête en janvier 1157 et interrogea plusieurs des hommes présents à l'assemblée mortuaire. Ces huit laïcs déclarèrent avoir vu Bérenger « dans les derniers jours de sa vie » tout donner à ses fils y compris les dîmes et les revenus ecclésiastiques. Pour soutenir leur déposition et la renforcer par un effet de réel, ils affirment qu'alors Bérenger leva solennellement le doigt : sursum in

\footnotetext{
${ }^{10}$ En 844, Charles le Chauve confirme à des Hispani du Biterrois leur possession des villae d'Aspiran et de Lignan (HGL, II, Preuves, col. 228) ; en 881, Carloman à Rainard les deux villae et d'autres biens (HGL, V, col. 71); en 897, Rainard vicomte cède les deux villae à l'évêque de Béziers (HGL, vol. V, col. 92). Voir la monographie sur Thézan dans C. DUHAMEL-AMADO, Genèse des lignages méridionaux, tome II, à paraître (La famille aristocratique languedocienne, thèse dactylographiée, Paris IV, 1995, tome II, livre 2, p . 427-453, spécialement aux p. 436-437).

${ }^{11}$ Église disparue de la commune de Montblanc (canton de Servian, Hérault, à14 km au nord-est de Béziers). Elle devait se situer vers le sud de l'actuel village, vers le domaine de Coussergues.

${ }^{12}$ O. TERrin éd., Cartulaire du Chapitre d'Agde, Nîmes, 1969, acte n 11 , p. 22.

${ }^{13}$ Corroboration faite au mois de décembre de la même année 1185, au castrum de Saint-Pons de Mauchiens, en présence de Pierre de Corneilhan, archidiacre, de Raimond de Bessan, chanoine, d'Ade (ou Adam) chapelain de Sainte-Marie et Saint-Pons, de Michel et de Pierre Brunus, prêtres et de Benedictus, diacre (mention dorsale). Au parchemin étaient donc appendus deux sceaux, celui de l'évêque d'Agde (perdu) et celui de Roger II dont il est question plus loin.

${ }^{14}$ Nézignan-l'Évêque, commune du canton de Pézenas (Hérault). Le nom moderne est évocateur : le castrum est entièrement aux mains de l'évêque d'Agde (Cartulaire du Chapitre d'Agde, op. cit., n³52 : confirmation d'un diplôme de Charles le Chauve par Louis VII en faveur de l'évêque d'Agde, qui lui accorde, entre autres, totum castrum de Nasignano pro alodio cum toto ejus territorio, p. 327).
} 
digito $^{15}$. Il s'agit du même rituel de donation que dans notre charte, un de ces «jeux de mains » qui scandent les cérémonies et les ancrent dans la mémoire des assistants ${ }^{16}$.

Si Guillelma est inconnue par ailleurs, Pierre en revanche est mentionné dans plusieurs autres actes, dont un qui le qualifie de miles pour le distinguer d'un autre Pierre de Thézan, clericus $^{17}$. Mais c'est surtout son seigneur, Bérenger de Thézan, qui est bien connu. Il est issu d'une des plus importantes familles de la plaine biterroise, qui fut sans doute alliée aux $\mathrm{IX}^{\mathrm{e}}-\mathrm{X}^{\mathrm{e}}$ siècle avec les comtes de Toulouse, puis qui appartint au proche entourage des vicomtes de Béziers à la fin du $\mathrm{X}^{\mathrm{e}}$ et au début du $\mathrm{XI}^{\mathrm{e}}$ siècle $^{18}$. Quand les Trencavel, vicomtes d'Albi à l'origine, prirent possession de l'héritage de ces premiers vicomtes biterrois dans les années 1060 , ils furent toujours en butte à l'opposition de l'ancienne aristocratie de la plaine de Béziers, d'origine wisigothique et très liée aux vicomtes de Narbonne et à l'évêché. La liste des témoins de notre acte est à cet égard éclairante ; sont présents des membres de cette haute aristocratie qui gravite autour de l'évêque : Bérenger de Lignan - qui se porte garant du prélat biterrois dans un accord en $1194^{19}-$, ou Guilhem d'Abeilhan, seigneur de Pézenas. La liste comprend aussi des proches du seigneur de Thézan : son parent Bermond de Thézan et Bérenger de Boujan. Pour le vicomte Trencavel, le terrain est donc plus ou moins hostile. Roger II n'est d'ailleurs pas présent en personne à la transaction et il n'intervient pas directement dans le document. Comment alors expliquer la présence de son sceau au bas de la charte ? C'est que le vicomte s'était fait représenter à cette alliance hautement stratégique entre un vassal de grands seigneurs biterrois et une fille issue de membres de l'entourage des évêques d'Agde. Un certain Pierre Isarn souscrit la charte, il se dit «bayle du seigneur Roger », c'est-à-dire représentant du vicomte ${ }^{20}$. Mais surtout, le chirographe fut rédigé par Bernard Cota, scribe de la cour vicomtale de Roger à Béziers et garde du sceau vicomtal ${ }^{21}$.

La leçon à tirer de l'analyse de cette charte est donc paradoxale. Elle révèle la force des réseaux biterrois, leur ancienneté, leur solidarité ; à la fin du XII ${ }^{\mathrm{e}}$ siècle, ils ont rallié l'évêque contre le vicomte. Mais, bien que le vicomte soit en position de faiblesse, cette aristocratie a jugé bon de faire corroborer une de ses transactions de son sceau ${ }^{22}$. Le scribe vicomtal qui rédige et qui scelle joue donc le rôle d'une chancellerie, enregistrant un acte privé sous un

\footnotetext{
${ }^{15}$ La charte qui relatait cette enquête est perdue, mais a été traduite, apparemment fidèlement, par un érudit de la fin du XVIII ${ }^{\mathrm{e}}$ siècle : Chérin, p. 2, verso. Tous ces renseignenements sont issus de la thèse dactylographiée de C. Duhamel-Amado (op. cit., tome 2, livre II, p. 443-444). Il est aussi à souligner que l'on a conservé pour le château et la seigneurie de Thézan un important fonds d'archives laïques : F. PASQUIER et S. OLIVE, Fonds Thézan aux Archives du château de Léran, Lauriol, Montpellier, 1911.

${ }^{16}$ Voir en dernier lieu R. JACOB, «Bannissement et rite de la langue tirée au Moyen Age. Du lien des lois et de sa rupture », Annales HSS, septembre-octobre 2000, p. 1039-1079 (spécialement p. 1051-1054, sur le rôle du doigt levé).

${ }^{17}$ Cartulaire du Chapitre d'Agde, $\mathrm{n}^{\circ} 145$, p. 144 (1202). Il abandonne en compagnie de ses deux fils, Pierre et Guilhem Jordan, des dîmes à Coussergues (ibidem, n²22, p. 205, sans date, début du XIII ${ }^{\mathrm{e}}$ siècle).

${ }^{18}$ Sur tout cela, démonstration et références dans C. DUHAMEL-AMADO, op. cit., Monographie Thézan.

${ }^{19}$ En 1194, Bérenger de Lignan se porte garant de l'évêque dans une sécurité réciproque avec Bertrand de Saissac qui agit comme tuteur lors de la minorité du jeune vicomte Raimond Roger, après la mort de Roger II (HGL, vol. VIII, col. 429).

${ }^{20}$ Il peut s'agir de Pierre Isarn de Lombers ou de Pierre Isarn de Castres (tous deux sont mentionnés dans l'entourage de Roger II en ces années). En tout cas, son nom le rattache à l'aristocratie albigeoise. À la cour des Trencavel, le bayle apparaît habituellement dans un contexte domanial, par opposition au viguier qui est en charge de la justice, de l'ordre public ou du contrôle des châteaux.

${ }^{21}$ Bernard Cota était aussi scribe public de la ville de Béziers, investi par le vicomte et l'évêque (HGL, vol. VIII, col. 348, en 1180); il agit cependant ici en tant que scribe vicomtal. Sur la nomination du scribe public à Béziers, voir H. DÉBAX, «Un cartulaire, une titulature et un sceau », loc. cit.

${ }^{22}$ Le recours à une corroboration vicomtale peut aussi être motivé par les changements sur le siège épiscopal biterrois. En 1185 en effet meurt l'évêque Bernard, de la lignée castrale de Lunel ; lui succède Gaufred de la famille vicomtale de Marseille. La vacance d'autorité dans l'évêché de Béziers a certainement poussé les contractants à avoir recours au pouvoir vicomtal.
} 
sceau public pour garantir la teneur de la charte et l'exécution des engagements pris par les deux parties. En Languedoc, des sceaux sont attestés depuis le milieu du XII ${ }^{\mathrm{e}}$ siècle, mais il s'agit ici d'une des premières attestations d'un tel usage d'un sceau de juridiction ${ }^{23}$.

La seconde charte, beaucoup plus simple et courte, donnera lieu à un commentaire plus succinct. La présence du sceau vicomtal s'explique ici de manière directe : l'acte atteste que Raimond Roger, vicomte, fils de Roger II, fit une concession à la commanderie templière de Pézenas, un lundi de février 1202 (fig. 3, en annexe). Raimond Roger, avec l'accord de membres de son proche entourage, Étienne de Servian ${ }^{24}$, Bernard Pelapol ${ }^{25}$ et Jourdain de Saint-Félix ${ }^{26}$, octroya aux Templiers le privilège de voir toutes leurs causes jugées à la cour vicomtale. La charte fut solennellement proclamée dans le castrum-même de Pézenas, audessus de la citerne, en présence de Jourdain de Saint-Félix qui devait être le représentant du vicomte, d'un bayle nommé Raimond et de quatre petits seigneurs locaux, inconnus par ailleurs. Elle fut écrite par un certain Poncius Borellonus, sous la dictée d'un notaire de Pézenas nommé Pierre le chapelain.

Les bénéficiaires sont les frères des «maisons de la milice» (du Temple), représentés par Frotard de Roquessels ${ }^{27}$, précepteur de la maison de Périès ${ }^{28}$, et Guilhem d'Auvergne ${ }^{29}$, précepteur de la maison de Pézenas. Ce précepteur des Templiers de Pézenas reçut par ailleurs, en ce même mois de janvier 1202, donation des dîmes de Coussergues ${ }^{30}$, église très proche de Trémoulet : des droits enchevêtrés sur ces deux paroisses expliquent sans doute l'insertion de la première charte analysée plus haut dans le chartrier des Templiers - puis dans le fonds de Malte.

L'acte scellé par Raimond Roger est intéressant à plus d'un titre. Il révèle tout d'abord l'orientation privilégiée des dévotions dans l'aristocratie languedocienne pour les Ordres militaires, et spécialement pour les Templiers. Dès leurs débuts, ceux-ci bénéficièrent du soutien appuyé d'un certain nombre de lignages seigneuriaux - comme ceux de Barbaira et de Trèbes à l'origine de la fondation de la commanderie templière de Douzens en Carcassès, de Roger I ${ }^{\text {er }}$ (vicomte Trencavel) qui élit sépulture dans une maison templière en 1150, de Guilhem de Tortose, frère de Guilhem VII de Montpellier, qui mourut dans la maison des Templiers de Montpellier vers 1157, de Raimond V, comte de Toulouse, qui prit sous sa

\footnotetext{
${ }^{23}$ Les comtes de Toulouse utilisent une bulle qui fait office de sceau de juridiction dans leurs domaines melgoriens et rhodaniens à partir de 1180, et la pratique est également répandue dans le Rouergue de la fin du $\mathrm{XII}^{\mathrm{e}}$ siècle (E. G. LÉONARD, Catalogue des actes de Raymond V de Toulouse, Nîmes, 1932, p. LXVIII et p. LXXI ; M. DE Framond, Sceaux rouergats du Moyen Age, Rodez, 1982, p. 37).

${ }^{24}$ Héritier d'une très importante famille de l'aristocratie castrale liée aux vicomtes de Narbonne, il semble avoir fait le choix de la fidélité aux Trencavel à la fin du XII et au début du XIII ${ }^{\mathrm{e}}$ siècle : il reçut de Raimond Roger une autorisation de fortifier le podium de Valros en 1199 ( $H G L$, vol. VIII, col. 453). Il fut accusé d'hérésie et Simon de Montfort le priva de tous ses biens en 1212.

${ }^{25}$ Seigneur du Carcassès qui fut viguier en Biterrois pour les Trencavel de 1199 à 1206.

${ }^{26}$ Saint-Félix de Lauragais ; plusieurs membres de la famille seigneuriale furent viguiers du Carcassès pour les Trencavel (Jourdain lui-même est cité comme viguier en 1190). Sur ces trois personnages, voir C. DuHAMELAMADO, "L'entourage des Trencavel au XII ${ }^{\mathrm{e}}$ siècle", Les voies de l'hérésie. Le groupe aristocratique en Languedoc (XII ${ }^{e}$-XIII ${ }^{e}$ siècles), colloque de Couiza 1995, Collection Heresis nº 8 , Carcassonne, 2001, p. 34-35.

${ }^{27}$ Roquessels, tout près de Faugères (canton de Roujan). Il est sans doute apparenté à l'évêque de Béziers Guilhem de Roquessels (1199-1205), qui fut déposé en février 1205 puis assassiné par ses serviteurs en avril.

${ }^{28}$ Peiroi dans le texte : il s'agit de Périès (hameau de la commune de Nissan-lez-Ensérune, canton de Capestang dans l'Hérault), «la plus importante maison de l'ordre du Temple dans le Narbonnais » (E. BONNET, «Les maisons de l'ordre du Temple dans le Languedoc méditerranéen », Cahiers d'histoire et d'archéologie, 9, 1936, p. 170 ; cité par F. HAMLIN, Toponymie de l'Hérault, Montpellier, 2000, p. 287).

${ }^{29}$ La graphie n'est pas sûre Alvernia ou Alumnia ; même hésitation sur son nom dans l'édition de O. Terrin pour la charte mentionnée à la note suivante.

${ }^{30}$ Cartulaire du chapitre d'Agde, n 422 , p. 406 ; un mercredi de janvier 1202. La donation est faite par Sibilde, héritière de la lignée castrale de Murviel, et confirmée par sa sœur Tiburge.
} 
protection les Templiers de Saint-Gilles, etc. Ils y trouvaient certainement des pratiques conformes à leurs idéaux chevaleresques et un moyen de contourner une Église officielle qui ne les satisfaisait plus ${ }^{31}$. Dans notre charte, Raimond Roger ne fait d'ailleurs que confirmer une concession déjà effectuée par son père, Roger II.

En outre, cette charte révèle l'ampleur prise par la renaissance du droit romain en Languedoc, à la fin du $\mathrm{XII}^{\mathrm{e}}$ et au début du XIII ${ }^{\mathrm{e}}$ siècle. Raimond Roger, comme son père l'avait fait auparavant, a pu assurer les Templiers qu'ils seraient assistés à sa cour de legendarii et de causidici literati ${ }^{32}$, c'est-à-dire de juristes et d'avocats lettrés. De tels personnages apparaissent en Languedoc à partir du milieu du $\mathrm{XII}^{\mathrm{e}}$ siècle. Les mentions les plus précoces sont à trouver à Montpellier ${ }^{33}$, dans l'entourage de l'évêque et des chanoines d'Agde ${ }^{34}$, à Melgueil (prieuré Saint-Jacques, dépendant de l'ordre de Saint-Ruf d'Avignon), à Maguelone. Ces juristes s'intitulent couramment magister, doctor, jurisperitus, causidicus ; ils forment dans la seconde moitié du XII ${ }^{\mathrm{e}}$ siècle un milieu intellectuel où circulent les textes et les idées ${ }^{35}$. Le terme de causidicus se rencontre couramment dans les chartes pour désigner les avocats, et l'adjectif literatus semble bien recouvrir une précision technique : il s'agit d'un avocat gradué en droit, qui a fait des études juridiques ${ }^{36}$. Legendarius semble en revanche être un hapax. Les dictionnaires de latin médiéval n'enregistrent en effet pour ce substantif qu'un sens fort éloigné de «recueil de vies de saints ${ }^{37}$. On ne peut donc que proposer des conjectures: legendarius serait employé pour désigner une personne ayant suivi un enseignement, quelqu'un qui sait lire, mais plus précisément ici qui a aussi des connaissances minimum en droit (un équivalent de legisperitus ?).

\section{Les sceaux}

La première empreinte conservée est celle de Roger II, vicomte de Béziers, de Carcassonne, de Razès et d'Albi (1167-1194). De forme ronde, ce sceau de cire brune est fragmentaire : seul un tiers supérieur est conservé. Son diamètre peut être restitué et estimé à environ $70 \mathrm{~mm}$ selon le rayon de courbure du bord de l'empreinte. Le mode d'apposition adopté est classique : le sceau est pendant, ses attaches sont constituées de larges lanières de cuir épais fixées par un nœud sur un repli de parchemin ${ }^{38}$. Ce dernier porte également la trace d'un scellement disparu, correspondant à celui de Pierre Raimond, évêque d'Agde (1173$1191)^{39}$.

\footnotetext{
${ }^{31}$ Voir M. BouRIN, «Autour des ordres militaires : des relents d'anticléricalisme ?», L'anticléricalisme en France méridionale (milieu XII -début XIV siècle), Fanjeaux 38, 2003, p. 252-253.

${ }^{32}$ Voir Du Cange et Niermayer s.v.

${ }^{33}$ Mention très précoce d'un certain Dulcianus dès 1122 (A. GOURON, «Les plus anciens testaments français », réimpr. Droit et coutume en France, Variorum, 1993, III, p. 288-289), puis bien entendu Rogerius et Placentin (Id, « Maîtres français et renaissance juridique du XII ${ }^{\mathrm{e}}$ siècle », réimpr. Droit et coutume en France, Variorum, 1993, XV).

${ }^{34}$ Vers 1154, Rotbertus doctor (P. CHASTANG, Lire, écrire, transcrire. Le travail des rédacteurs de cartulaires en Bas-Languedoc (XI ${ }^{e}$-XIII ${ }^{e}$ siècles), Paris, CTHS, 2001, p. 289 et suivantes, spécialement 300-301).

${ }^{35}$ A. Gouron, «Comment dater la venue de Placentin à Montpellier? », réimpr. Droit et coutume en France, Variorum, 1993, IV, et plus généralement tout le volume. Voir aussi : La science du droit dans le Midi de la France au Moyen Age, Variorum, 1984 et Études sur la diffusion des doctrines juridiques médiévales, Variorum, 1987.

${ }^{36}$ Sur les deux catégories d'avocats, litterati et non litterati (au milieu du XIII ${ }^{\mathrm{e}}$ siècle): G. GIORDANENGO, «Jean Blanc, feudiste de Marseille», réimpr. Féodalités et droits savants dans le Midi médiéval, Variorum, 1992, IX, 73-74 et Retractationes, p. 8.

${ }^{37}$ J. F. NIERMAYER, Lexikon..., s.v. legendarius. Du Cange ne fait pas figurer cette entrée.

${ }^{38}$ Le sceau a été imprimé perpendiculairement à la charte.

${ }^{39} \mathrm{La}$ mention hors teneur qui se trouve au dos de l'acte permet d'identifier le second sigillant.
} 
L'avers appartient au type équestre (fig. 4, en annexe). Dans le champ, Roger II est représenté en cavalier, assis sur une monture chevauchant vers la droite. Il est revêtu d'un haubert qui laisse apparaître son visage et il est coiffé d'un casque de forme sphéro-conique qui vient couper le haut de la légende du sceau. Il porte un long écu en amande, assez effilé, et à pointe arrondie. Cette protection non armoriée est retenu par la guiche qui passe sur son épaule gauche. De son bras droit replié, il tient couchée contre son flanc une lance dont on distingue une partie du pennon. Le tapis de selle de son cheval apparaît distinctement. Le champ du revers, de même type, est quasiment identique (fig. 5, en annexe). La légende, comprise entre deux cercles, est très mutilée. Néanmoins, elle peut être ainsi restituée sur les deux faces : /SIG[ILLUM ROGERII VICECOMITIS BITERR]IS. Enfin, la mention du scellement apparaît dans la formule de corroboration de l'acte : et ad majorem auctoritatem sigillo domini Rotgerii vicecomitis Biterris hec corroboravit.

La seconde empreinte, celle de Raimond Roger (1194-1209), fils du précédent vicomte, se trouve apposée à la charte de février 1202. L'empreinte est également très fragmentaire, la partie inférieure est brisée en deux morceaux mais elle se trouve encore retenue par l'attache. Le module de ce sceau rond est légèrement plus réduit que le précédent $(60 \mathrm{~mm})$. L'empreinte de cire naturelle, brun foncé, est pendante sur des cordelettes de chanvre tressées passant par deux incisions réalisées dans le repli du parchemin.

Comme son père, Raimond Roger a adopté un type équestre où on le voit également chevaucher à droite. Sur l'avers du sceau, son corps disparaît derrière un long écu triangulaire qui le protège (fig. 6, en annexe). Son bras droit, placé en arrière, brandit une épée à pommeau rond et à quillons droits ; l'arme est à deux tranchants avec une rainure centrale qui sert de gorge d'évidement. Le revers reprend le modèle du type équestre mais la partie conservée permet de mieux lire l'écu où demeurent des éléments héraldiques évoquant les premières armoiries des Trencavel, à savoir un fascé qui apparaissait sur l'empreinte de 1211 (fig. 7, en annexe). Le cheval, non houssé, est au galop. La pointe du fourreau de l'épée, le tapis de selle, l'étrivière et l'éperon sans molette de la jambe gauche du vicomte sont aisément perceptibles. La légende de l'avers, malgré les cassures partielles, peut être ainsi transcrite : [SIGILLUM RAIMUNDI RO]GERII VICECOM[ITIS] BIT[ERRIS]. Celle du revers reprend la même titulature : [SIGILLU]M RAIMUNDI ROG[ERI]I VICEC[OMITIS BITERRIS]. Quant à la formule de corroboration, elle annonce précisément le scellement vicomtal : In / tali vero pacto predictum donum domui milicie templi facio ut firmissimum / ac stabilissimum omni tempore permaneat et ut majorem firmitatem habeat sigillo / meo sigillari precipio.

La découverte de l'empreinte de sceau de Roger II est à mettre en relation avec la politique de prestige et d'affirmation développée par ce prince dans les années 1180 . La confection du cartulaire, l'adoption d'une quadruple titulature (même si le sceau n'en conserve qu'une seule ici), la réalisation d'une matrice ont lieu quasiment au même moment, vers $1185^{40}$. L'indéniable qualité esthétique de ce sceau montre que le vicomte et ses conseillers ont fait appel à un graveur expérimenté. La puissance de Roger se manifeste également à travers une représentation qui insiste sur son pouvoir de commandement symbolisé par la lance à pennon. Toutefois, l'usage du sceau demeure une décision prise tardivement : les Trencavel semblent en retard par rapport aux comtes de Toulouse qui scellent régulièrement depuis 1156 ou par rapport à de grandes familles seigneuriales languedociennes telles que les Uzès-Posquières, les Anduze, les Sabran qui font usage d'un sceau depuis 1174, voire depuis 1169 pour certains comme les sires de Calmont-d'Olt ${ }^{41}$. D'autres dynastes valident aussi leurs

\footnotetext{
${ }^{40} \mathrm{H}$. DÉBAX, La féodalité languedocienne, XI ${ }^{e}$-XII ${ }^{e}$ siècles. Serments, hommages et fiefs dans le Languedoc des Trencavel, Toulouse, 2003, p. 288-289.

${ }^{41}$ L. MACÉ, Les comtes de Toulouse et leur entourage, XIIe-XIII ${ }^{e}$ siècles. Rivalités, alliances et jeux de pouvoir, Toulouse, 2000, p. 295 et p. 323 ; M. DE FRAMOND, Sceaux rouergats du Moyen Age, Rodez, 1982, p. 24.
} 
actes autour de la seconde moitié du siècle : le comte de Rodez (1140), le comte de Provence (1150), le comte de Barcelone (1157), le comte de Forcalquier (1168). En revanche, le comte de Foix ne s'y résigne qu'à partir de 1188 ; les seigneurs de Montpellier et les vicomtes de Narbonne corroborent également leurs chartes seulement quelques années plus tard (1192 et $1193)^{42}$.

L'empreinte de sceau de Raimond Roger est, quant à elle, contemporaine de ses premiers pas dans l'exercice du pouvoir : âgé de 14 ans, il est majeur depuis avril $1201^{43}$. L'une de ses premières décisions fut donc la réalisation d'une matrice sigillaire qui lui soit personnelle. Par ailleurs, cette dernière permet de faire remonter l'attestation des armoiries vicomtales au tout début du XIII ${ }^{\mathrm{e}}$ siècle (1202). Le revers conserve les traces d'au moins deux fasces et deux figures sont perceptibles au toucher sur la fasce centrale de l'écu. Il est tentant d'y voir les armes traditionnelles des Trencavel, un écu composé de six pièces constituant un fascé d'hermine, tel qu'il apparaît sur une empreinte de juillet $1248^{44}$. Il serait hasardeux pour autant d'en accorder la paternité au jeune vicomte et à son entourage. L'empreinte de 1185 , très effacée, ne livre aucun relief à la surface de l'écu. Mais l'usure de la cire peut expliquer l'absence d'armes et n'autorise donc pas à conclure hâtivement que Roger II ne possédait pas d'armoiries ${ }^{45}$. Rappelons que la fonction première de l'usage héraldique est de permettre au combattant d'être identifié sur le champ de bataille ; les différentes campagnes militaires des Trencavel au cours de la grande guerre méridionale et leur propre réputation de grand pourfendeur (Trenca vel) ne pouvaient que les contraindre à adopter rapidement des signes de reconnaissance. Un écu sans armes aurait été un fait plutôt remarquable en cette fin de siècle. Était-ce déjà un fascé ? Le sceau de 1185 laisse la question ouverte.

\section{Transcriptions}

H Malte, Pézenas, 10
$\mathrm{ABC}$
DEF
GHI
KLM
NOP

In nomine domini anno a nativitate ejusdem $\mathrm{M}^{\circ} \mathrm{C}^{\circ} \mathrm{LXXX}^{\circ} \mathrm{V}^{\circ}$. Regnante rege Philippo, $\mathrm{V}$ idus decembris, fuit facta hec carta commutacionis honoris quam / facimus inter nos, ego Petrus de Tedano et ego Guillelma uxor ejusdem Petri. Ego itaque Petrus de Tedano per me et per meos, cum consilio et voluntate Berengarii de Tedano et / Ermessendis uxoris ejus de quibus teneo subscriptum honorem meum ad feudum, bona fide et sine dolo, cum hec carta dono, trado omninoque derelinquo nunc et in / perpetuum titulo perfecte et perpetue commutacionis sine ulla mea meorumque retencione tibi predicte Guillelme uxori mee et tuis ad omnes voluntates vestras vestrorumque / perpetuo faciendas IIII $^{\text {or }}$ campos scilicet meos cum suis pertinenciis quos habeo in terminio de Aspirano. Quorum unus qui vocatur Campus Grassus affrontat ex circio in hono/re Berengarii de Tedano, ex aquilone in campo Michaelis

\footnotetext{
${ }^{42}$ M. DE FRAMOnd, op. cit., p. 116 ; M. FABRe, Sceau médiéval. Analyse d'une pratique culturelle, Paris, 2001, p. 34-35 ; M. OUdOT DE DAIMVILle, Les sceaux conservés dans les archives de Montpellier, Montpellier, 1952, p. 28 ; J. CAILLE, «Ermengarde, vicomtesse de Narbonne (1127/29-1196/97). Une grande figure féminine du Midi aristocratique », La femme dans l'histoire et la société méridionales, Narbonne, 1995, p. 25 n. 133.

${ }^{43}$ C. DUHAMEL-AMADO, art. cit., p. 33-34.

${ }^{44}$ DOUËT D'ARCQ, t. I, p. 384-385, n 761.

${ }^{45}$ Le cas est loin d'être rare. Par exemple, sur le revers d'une bulle de 1204 provenant de la chancellerie du roi Pierre d'Aragon, le souverain paraît chevauchant avec un écu où les fameux pals sont totalement effacés (Barcelone-Carcassonne : destins croisés de deux comtés (IX $X^{e}$-XIII ${ }^{e}$ siècles), catalogue d'exposition, Carcassonne, 1997, p. 48).
} 
de Aspirano, ex meridie in Taurono. Alius campus qui vocatur de Goro affrontat ex circio in campo Michaelis de / Aspirano, ex aquilone in honore de Berengarii de Tedano et Ugonis de Paulino, ex meridie in campo Raimundi Caballarii et Guillelmi Barania. Tercius campus est juxta /molendinum Guillelmi Barania et affrontat ex circio in honore ipsius Guillelmi Barania, ex meridie in honore Berengarii de Tedano, ex altano in campo Guillelmi Aitorii. / Quartus campus qui vocatur de Vas affrontat ex circio in via qua itur de Tedano ad Aspiranum, ex meridie in condamina episcopi Biterris, ex altano in honore ipsius episcopi. / Et* ego Guillelma dono similiter et trado et penitus derelinquo per me et per meos nunc et in perpetuum titulo perfecte et perpetue commutacionis sine ulla mea meorumque / retencione, cum consilio et voluntate Raimundi de Nasignano avunculi mei tibi Petro de Tedano marito meo et tuis ad omnes voluntates vestrorumque perpetuo faciendas / quicquid habeo et teneo vel habere debeo vel homo vel femina habet per me vel de me vel avus meus Raimundus de Nasignano vel ceteri predecessores mei visi sunt quoque / modo habuisse per se vel per alios et tenuisse in toto terminio et in parrochia sancti Andree de Tremoleto in hominibus seu feminis, in feudis seu in feudalibus, in domibus / in aquis, in pratis, in pascuis, in arboribus, in garricis, in terris, vineis, in cultis sive non cultis vel in omnibus aliis rebus. Ut autem hec omnia firma et illibata / maneant in perpetuum, ego Petrus de Tedano et ego Guillelma nos ambo simul per fides nostras promittimus et super hec sancta IIII ${ }^{\text {or }}$ evangelia corporaliter tacta jura /mus quod contra hanc commutacionem aliquo jure scripto vel non scripto vel aliqua occasione numquam veniemus nec venire faciemus sed eam semper ratam et firmam habebimus. / Hujus rei sunt testes Berengarius de Lignano, Raimundus de Nasignano, Guillelmus de Abiliano, Bermundus de Tedano, Berengarius de Boiano, Raimundus bajulus et Petrus Ysarnus ba/julus domini Rotgerii, Petrus Canabacerius, de fratribus templi Benedictus, Arnaudus Sutor. Jussu Petri de Tedano et uxoris ejus et Berengarii de Tedano et testium, Bernardus Cota scrip/sit in ecclesia sancti Stephani de Boiano et ad majorem auctoritatem sigillo domini Rotgerii vicecomitis Biterris hec corroboravit.

\section{*Un doigt pointé à la verticale est dessiné dans la marge de gauche et montre le Et du début de phrase de Guillelma}

Derrière, en haut de l'acte, rajout suivant :

Mandato et precibus Petri de Tesano et uxoris ejus Guillelme et Berengarii de Tesano, ego P. Ramundi Agathe episcopus homo imeritus hec ad perhenem rei memoriam sigillo meo sig/nari feci apud castrum castri Sancti Pontii anno et mense quo supra. In presentia Petri Agathe archidiaconi et Raimundi de Betiano Agathensis canonici et Ade ecclesie sancte Marie et sancti / Pontii capellani et Ugonis de Betiano et Michaelis sacerdotis et Petri Bruni sacerdotis et Benedicti diaconi.

H Malte, Pézenas, 19

In Christi nomine anno ejusdem incarnationis M CC II mense februarii, rege Philippo reg/nante. Ego Raimundus Rotguerius, gratie Dei vicecomes Biterris, bona fide et sine omni / dolo, cum hac presenti carta, nunc et in perpetuum irrevocabiliter, pro me et pro omnibus meis / successoribus et heredibus, voluntate et assensu Stephani de Cerviano et Bernardi / Pelapulli et Jordani de Sancto Felicio, concedo et dono amore Dei et redempcione / anime mee et patris mei et matris mee et omnium fidelium Christianorum sicut superius dic/tum est concedo et dono domino Deo et beate Marie et domui milicie templi et / tibi Frotardo de 
Rocosello, preceptori domus milicie de Peiroi et tibi Guillelmo de Alvernia / preceptori domus milicie de Pedenatio et omnibus fratribus ejusdem domus tam presentibus / quam futuris ut semper positis placitare et racionare placita vestra et causas vestras / in mea curia et in omnia (sic) patria mea cum legendariis et cum causidicis literatis. In / tali vero pacto predictum donum domui milicie templi facio ut firmissimum / ac stabilissimum omni tempore permaneat et ut majorem firmitatem habeat sigillo / meo sigillari precipio. Preterea ego Raimundus Rotguerius vicecomes predic/tus recognosco et cognosco quod pater meus Rotguerius dedit domui milicie / templi et fratribus ejusdem domus ut potuissent placitare et racionare omnia placita / sua cum legendariis et cum causidicis literatis in sua curia et in omni patria sua / et hunc eumdem donum laudo et concedo predicte domui milicie et fratribus ejusdem / domus pro me et pro omnibus meis successoribus et heredibus a modo in perpetuum. Hec carta fuit / laudata infra castrum de Pedenatio insuper cisternam, in presencia Jordani de / Sancto Felicio, Ramundi bajuli, Petri Ramundi de Baias, Petri de Salada, Si/cardi de Larocha, Bernardi de Gajano et Petri Capellani notarii de Pedena/tio qui hec dictavit et Poncius Borellonus mandato ejus et vicecomitis et aliorum / predictorum testium, feria IIa, hec omnia scripsit anno et mense quod supra.

Hélène DÉBAX Laurent MACÉ 
Fig. 1 : Charte de 1185, H Malte, Pézenas, 10 (cliché : Archives départementales de la Haute-Garonne)

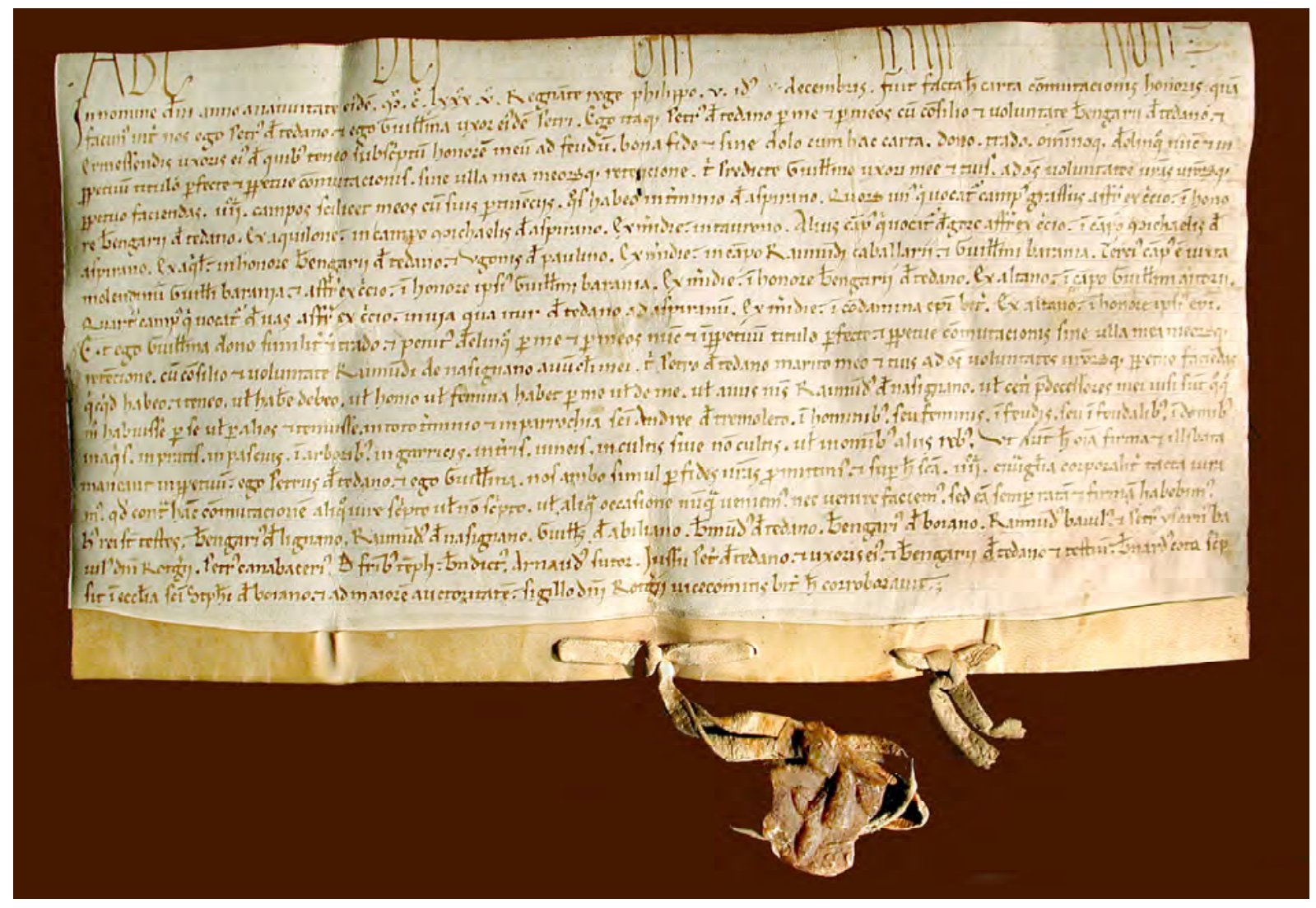

Fig. 2 : le doigt levé qui désigne « Et ego » 
Fig. 3 : Charte de 1202, H Malte, Pézenas 19 (cliché ADHG)




Fig. 4 : Avers du sceau de 1185 (cliché ADHG)

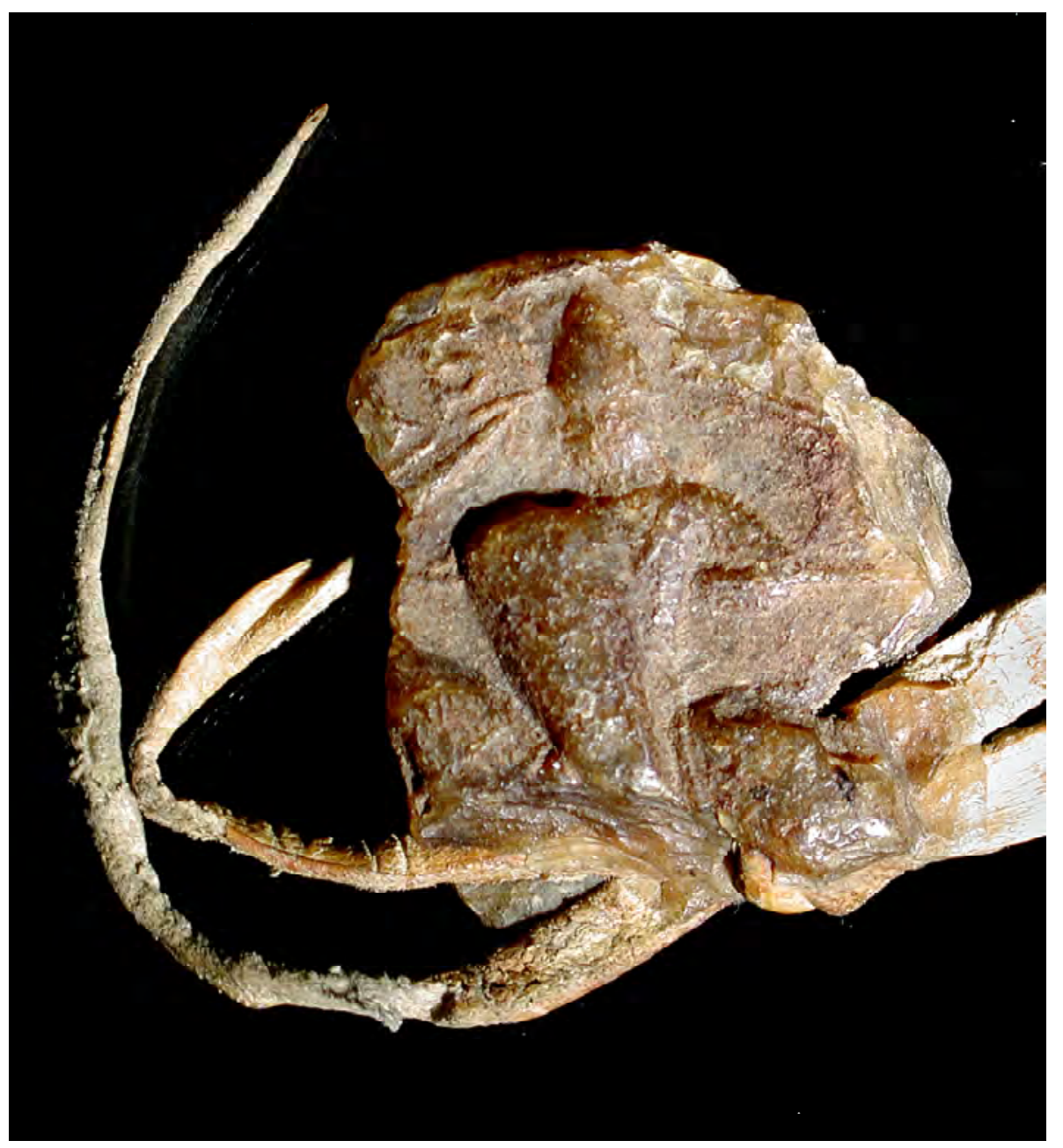


Fig. 5 : Revers du sceau de 1185 (cliché ADHG)

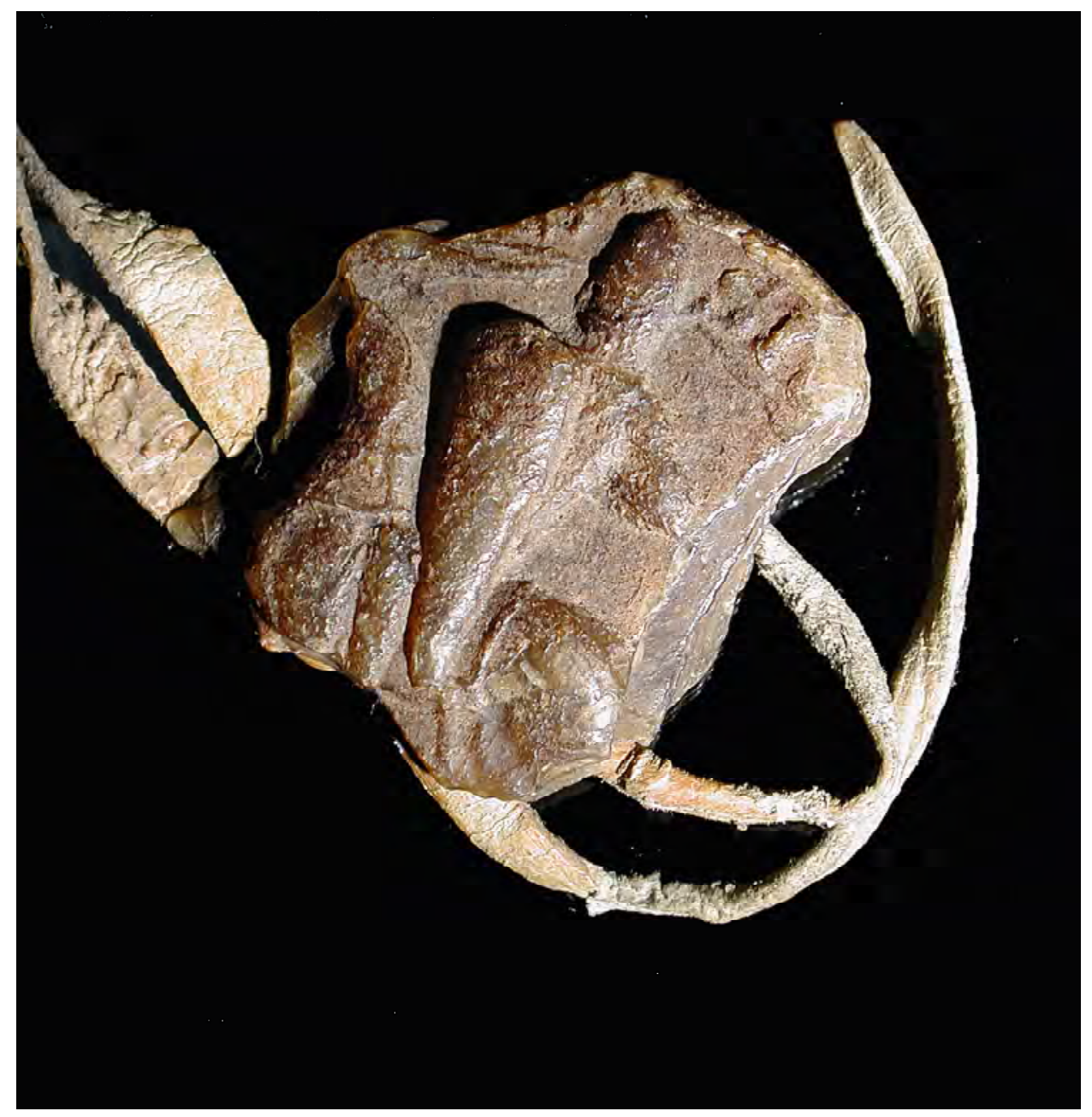


Fig. 6 : Avers du sceau de 1202 (cliché ADHG)

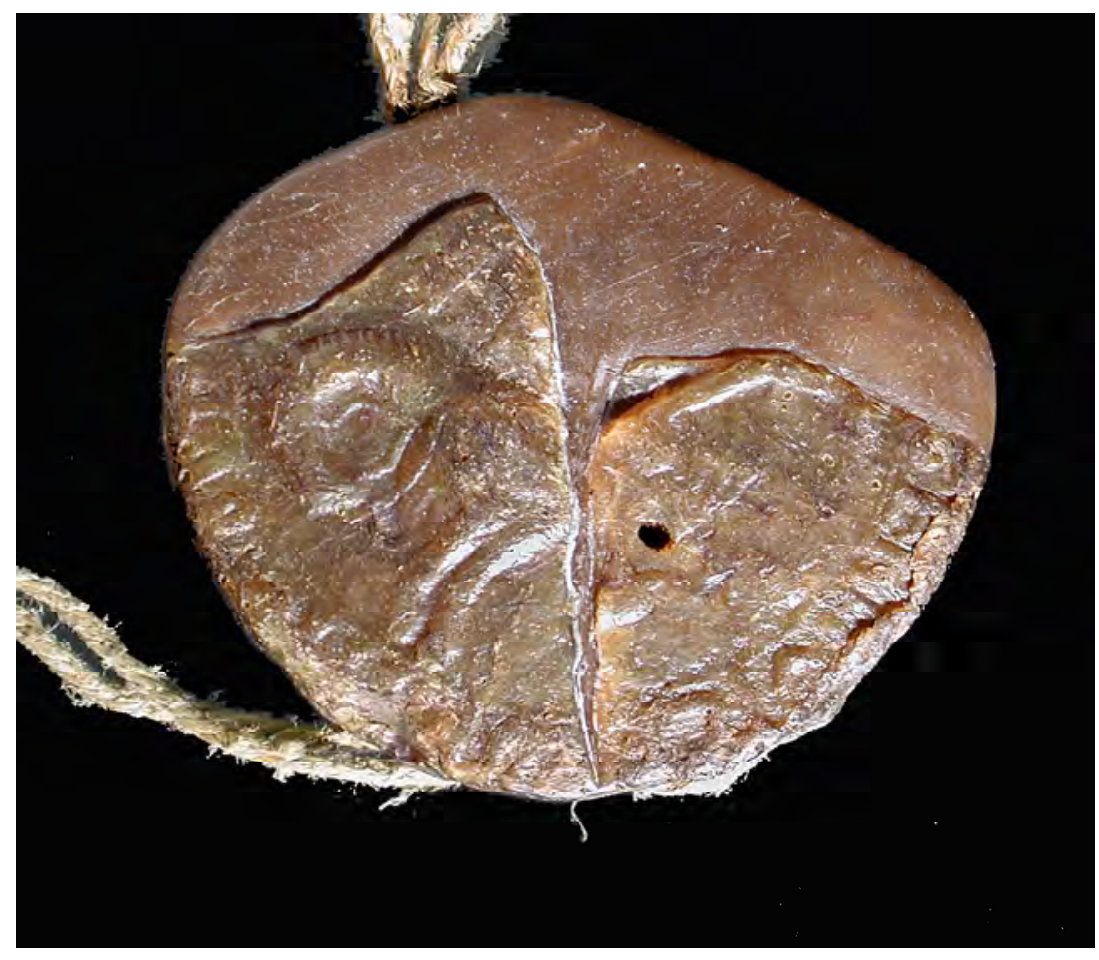


Fig 7 : Revers du sceau de 1202 (cliché ADHG)

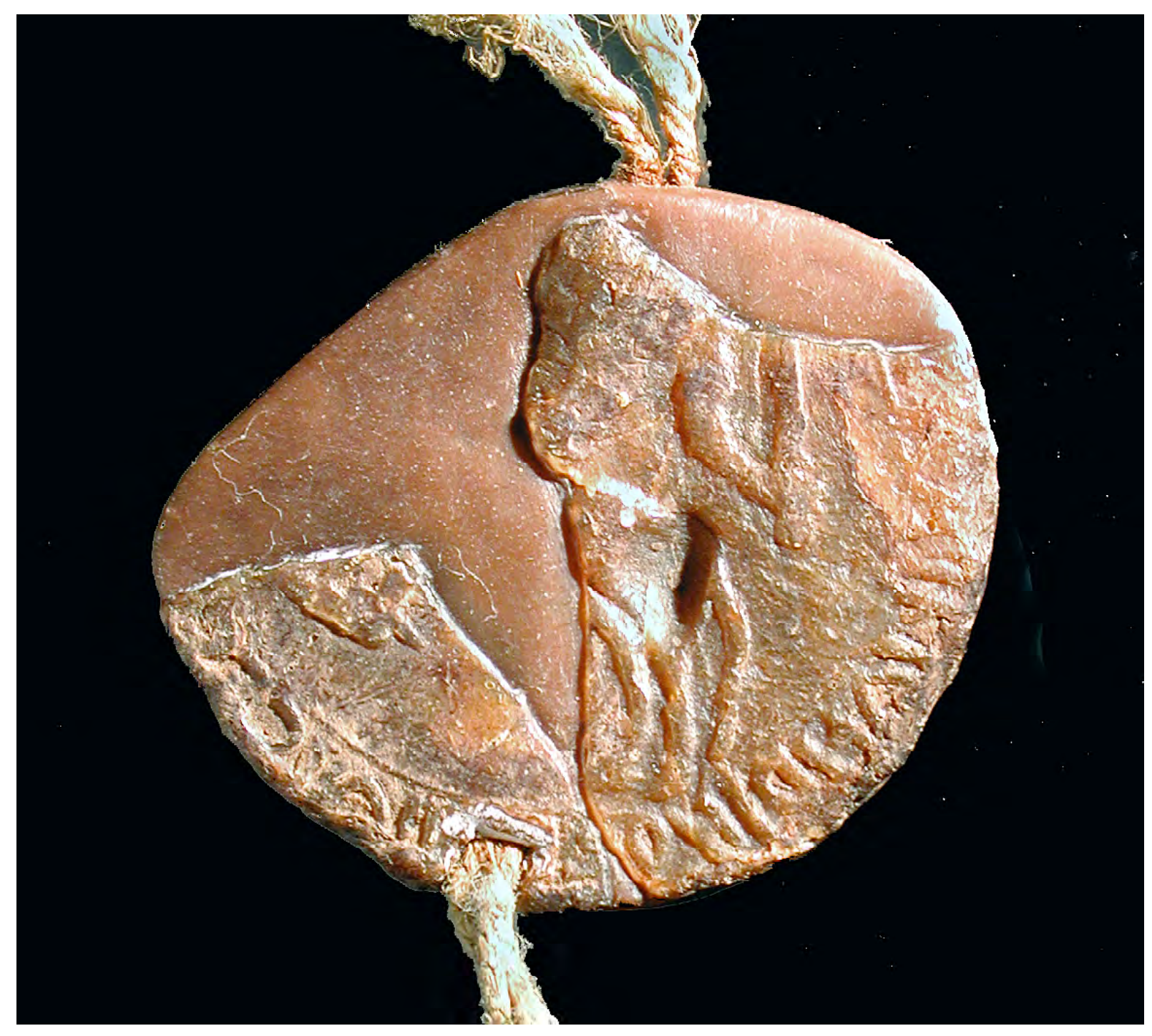

